\title{
Groundwork for Life Detection
}

A perspective contributed by The Center for Life Detection

\section{Primary Authors}

Tori Hoehler, NASA ARC (tori.m.hoehler@nasa.gov, 650-714-8601)

Will Brinckerhoff, NASA GSFC (will william.b.brinckerhoff@nasa.gov); Alfonso Davila, NASA ARC (alfonso.davila@nasa.gov); Dave Des Marais, NASA ARC (david.j.desmarais@nasa.gov);

Stephanie Getty, NASA GSFC (stephanie.a.getty@nasa.gov); Danny Glavin, NASA GSFC (daniel.p.glavin@nasa.gov); Andrew Pohorille, NASA ARC (andrew.pohorille@nasa.gov); \& Richard Quinn, NASA ARC (richard.c.quinn@nasa.gov)

\section{Co-Authors}

Lee Bebout, NASA ARC (leslie.e.bebout@nasa.gov); Jared Broddrick, NASA-ARC (Jared.t.broddrick@nasa.gov); Christopher Dateo NASA ARC (christopher.dateo@nasa.gov); Jennifer Eigenbrode, NASA GSFC (jennifer...eigenbrode@nasa.gov); Craig Everroad, NASA ARC (craig.everroad@nasa.gov); Fathi Karouia, NASA ARC/BMSIS (fathi.karouia@nasa.gov); Barbara Lafuente, NASA ARC/SETI Institute (blafuente@seti.org); Graham Lau, NASA ARC/ BMSIS (grahamlau@bmsis.org); Paul Mahaffy, NASA GSFC (paul.r.mahaffy@nasa.gov); Marc Neveu, NASA GSFC/University of Maryland (marc.f.neveu@nasa.gov); Andro Rios, NASA ARC/BMSIS (andro@bmsis.org); \& Sanjoy Som, NASA ARC/BMSIS (sanjoy@bmsis.org) 
Summary: This white paper frames life detection objectives as a systematic progression in our understanding of planetary environments and, within that framing, identifies a suite of research, technology development, and community activities that will advance our readiness to search for evidence of life beyond Earth.

\section{Introduction}

Ours could realistically be the generation that discovers evidence of life beyond Earth. We occupy this privileged position because the advancement of planetary sciences, astronomy, and biology has now made it possible to address the question "Are we alone?" with scientific rigor. NASA has adopted this civilization-level challenge as part of its charter. The scientific research and technology development communities must now work together to ensure that, should evidence of life exist within reach of our spacecraft and telescopes, we optimize the potential to find it.

There is much that NASA can do on the ground, now, to prepare more fully for forthcoming opportunities to seek evidence of life beyond Earth. The purpose of this white paper is to identify a suite of research and technology development activities, and frame the community-level dialog, that will lead to a rigorous scientific foundation for life detection efforts. While NASA has historically supported many of these activities, their collective bearing on life detection has not been fully considered, and there is a potential synergy among them that will be most fully realized if they are pursued as complementary components of a well-coordinated whole.

\section{A Framework for the Life Detection Endeavor}

The search for evidence of life risks being perceived as a departure from the traditional objectives of planetary science - an all-or-nothing 'swing for the fences' that stands to deliver little beyond the 'yes' or 'no' of its primary objective. This need not and should not be the case. Instead, this search can be pursued as part of a systematic progression in our understanding of planetary environments. Such a progression should encompass environmental characterization at every step, including the ultimate one: It is essential that any potential evidence of life, or a null result, be evaluated and understood within the context of the environment that hosts it. As such, a wellconceived arc of life detection activities will serve to expand our knowledge of planetary environments, whether evidence of life is found or not. Thus, the search for evidence of life is not a departure from the traditional objectives of planetary sciences but, rather, their logical extension into the next decades of planetary exploration. The Committee can promote the broader acceptance of life detection objectives by casting them in such light.

The progression of planetary science objectives within an astrobiological reference frame could be articulated as a set of questions, and associated observing strategies, that shift from general to specific and from inclusive to stringent.

\begin{tabular}{ll|l|}
\hline \multicolumn{1}{|c|}{ The path from comparative planetology to comparative biology } & Objectives \\
- & Questions & Comparative Planetology \\
- Could the environment support life as we know it? & Habitability \\
- What is the likelihood that life could originate in the environment? & Detectability \\
- If life is present in the environment (how) could we detect it? & Life Detection \\
- Are there indicators of life in the environment? & Comparative Biology \\
\hline
\end{tabular}


In this progression, "life detection" is not a singular objective but a range of possible observing strategies in which the relative balance of inclusivity vs. stringency should vary on the basis of the existing understanding of the target body. In this view, an observing campaign need not yield definitive evidence of life to have high value in the overall life detection endeavor. Instead, the emphasis is on complementarity of contextual, inclusive, and stringent measurement approaches and the importance of continuity between them. Competed mission classes have excellent potential to contribute at multiple points along this continuum, and NASA should tap the full extent of the community's creativity to realize this potential.

In the specific context of life detection, inclusivity seeks to minimize the probability of false negative results, while stringency seeks to minimize the probability of false positive results. We perceive the probability of false negative results as depending on the prevalence (occurrence rate) of a targeted observable across all biological systems and its detectability based on both signal strength (abundance, frequency, rate, etc.) and measurement capabilities. In parallel, the probability of false positive results depends on the prevalence and signal strength of both abiotic features and terrestrial contaminants that may mimic or obscure features sought as evidence of native biology.

The approach described above highlights a suite of research and analysis, technology development, and standardization activities that, if pursued in coordinated fashion, will optimize our potential to pursue life detection objectives through a balance among contextual, inclusive, and stringent measurement approaches.

\section{Standardization}

The diversity of potential biosignatures requires that community-level efforts be pursued to establish a standardized, evaluative framework that supports apples-to-apples assessment of their utility in addressing life detection objectives.

The NASEM report, An Astrobiology Strategy for the Search for Life in the Universe, recommended that, "NASA should support the community in developing a comprehensive framework for assessment - including the potential for abiosignatures, false positives, and false negatives - to guide testing and evaluation of in situ and remote biosignatures." (p.80)

The range of potential measurement strategies for addressing life detection objectives is large, diverse, and in many cases novel relative to those traditionally employed in planetary sciences. The language and standards used to demonstrate the "value" of a particular life detection approach can be similarly variable and particular to each approach. This may create the challenge of applesto-oranges comparisons in assessing the relative effectiveness of differing approaches. A comprehensive framework can help to standardize the basis on which the utility of diverse approaches is assessed, and the type of information that is sought and conveyed in proposing new potential biosignatures. The most critical element of such a framework is the selection of a set of uniformly applied "criteria" that represent a common basis for assessing utility in the context of life detection. This selection should result from dialog across a broad cross-section of the relevant science and technology communities. In keeping with the recommendation of the 2018 NASEM report, we propose, as a starting point for this dialog, that the factors influencing the probability of false positives and false negatives - specifically, the prevalence and signal strength of potential biosignatures, abiotic mimics, and Terrestrial contaminants - be considered as a draft set of criteria. We propose that such a framework forms the basis for a functional system that promotes and centralizes the exchange of information as life detection science and technology evolve. The format of such a system should ideally support: 
- Use in program planning, science definition, $\mathrm{R} \& \mathrm{~A}$, technology development, and education

- Clear traceability of high-level, summary, or evaluative content to the complete body of knowledge (e.g., as peer-reviewed reports) on which it is based

- Documentation of community commentary and debate regarding that body of knowledge

- User-contributed content, and flexibility to incorporate new information as life detection knowledge and measurement technology evolve

- Ready comparison between measurement requirements and existing and emerging measurement technology and capabilities

- A process for evaluation of potential biosignatures via uniformly applied criteria

Initiating this process and the associated community-level dialog now can serve to promote a vigorous before-the-fact dialog on standards of evidence and support an ability to balance contextual, inclusive, and stringent measurement approaches in addressing life detection objectives. Moreover, it can serve to identify critical areas for support - e.g., as promising but understudied potential biosignatures or measurement technology/capability needs - to most effectively advance readiness for life detection. The Committee can enable this process by reiterating the importance of creating a standard framework for assessing the traceability and utility of potential biosignatures to specific life detection objectives.

\section{Research and Anaylsis}

\section{Studies in origins of life, detectability, universal biosignatures, and astromaterials characterization will inform a strategy that balances contextual, inclusive, and stringent measurements in pursuit of evolving life detection objectives.}

The systematic progression in life detection objectives advocated here, and the proposal for "criteria" related to the probabilities of false negative and false positive results, identify several areas of R\&A that will be important in laying the groundwork for life detection in flight. We do not intend the following as an exhaustive list of research areas that support life detection objectives. Rather, we seek to identify areas whose specific bearing on life detection may be important but less clear, along with existing areas of emphasis where next steps are important to take.

\section{Origins of Life}

In the context of life detection, studies on the origins of life can extend our existing concept of habitability. As presently conceived in planetary sciences, habitability is concerned with whether the conditions in a given environment could support life as we know it - that is, cellular life that has diversified into a range of niches and evolved a wide range of capabilities and tolerances. Origin of life studies explicitly address the bearing of environment on life's emergence and the alternative trajectories of molecular and biochemical evolution that may be possible. This can serve to inform life detection approaches by:

- Constraining the conditions and processes required for life's emergence and estimating the likelihood that they are met at a given target. Because these conditions may be narrower or different than those required to support extant life, this may constitute an added 'screen' to help identify priority targets from among a range of nominally habitable environments.

- Identifying potential biosignatures that would specifically indicate non-Terrestrial origins. Such 
features, which might result from alternative trajectories of chemical evolution, would mitigate the potential for interference or false positives associated with Terrestrial contamination and indicate an independent genesis of life.

- Identifying potential biosignatures that are highly prevalent or conserved across biological systems or are associated with specific environmental conditions. This would aid in the design of measurement strategies that minimize the probability of false negative results.

- Determining the complexity of chemistry that might exist in the absence of life. This line of research considers the potential for barriers that halt the progress of molecular evolution at a complex but not yet animate level. This work will help in assessing the potential range of life-like abiotic chemistry that could increase the probability of false positive results.

\section{Detectability Science}

The presence of life does not guarantee an ability to detect it. The heterogeneous distribution of life on Earth vividly demonstrates that biological potential can vary by orders of magnitude along multiple axes - abundance, productivity, diversity, and others - as a function of multiple environmental factors. The evidence of that potential comes in a variety of forms, which are differently subject to modification by processes of transport, concentration/dilution, and preservation/degradation that can also range widely across environments. We should expect similar variability within and among potential targets of life detection missions. Measurement capabilities also differ by approach so that, overall, the probability of detecting of evidence of life in an inhabited system may vary substantially as a function of environment, potential biosignature sought, and measurement approach/technology employed. We address technology development in the following section and focus here on the need to understand how environmental context influences the potential quality and quantity of evidence for life. We can further distinguish the bearing of environment (e.g., as processes controlling the flux of resources) on the nature, abundance, diversity and productivity of life (biological potential) and subsequently on the modification of signals produced by life (biosignature potential).

On the environmental side, the objective of 'detectability science' is to develop an intellectual framework that combines spacecraft observations with an understanding of Terrestrial biology in its environmental context to aid in prioritizing next steps in R\&A, technology development, and development of optimal observing strategies in support of life detection objectives. In particular, detectability studies can inform instrument performance and measurement requirements, and identify precursor and context measurements that seek to minimize or assess the probability of false negative results. Detectability science strongly advances the traditional objectives of planetary environment characterization through its required focus on constraining the planetary processes that govern both the flux of resources to life and the transport and modification of potential biosignatures.

\section{Universal Biosignatures}

The probability of false negatives is influenced not only by the detectability of potential biosignatures, but also by their prevalence - that is, frequency of occurrence - in biological systems overall. Specifically, the potential exists to overlook life that does not produce the particular features targeted by a given measurement strategy. For this reason, we propose biological prevalence as one of the primary criteria by which potential biosignatures are assessed, and the essential one in developing inclusive measurement strategies.

Research on 'universal biosignatures' is an emerging area in which researchers seek to identify attributes that are highly prevalent or even universally present across biological systems. The 
research and technology development needed to identify and develop such 'universal' or 'agnostic' biosignatures are described in the white paper "Towards a more universal life detection strategy" (Chou, Greffenstette, et al.). Here, we add that it is important for research in this area to include an assessment of candidate universal biosignatures with respect to additional evaluation criteria, such as detectability and the prevalence of corresponding abiotic mimics. Similarly, universal biosignatures are, by definition, subject to interference from Terrestrial contamination. Therefore, we should seek to identify specific strategies to mitigate, rule out, or control for this possibility.

\section{Astromaterials}

Any unambiguous life detection discovery will require that all plausible abiotic formation mechanisms for the potential biosignature(s) be ruled out. The organic matter in meteorites, IDPs, and samples returned from asteroids and comets has been studied with a focus on understanding its potential to supply the raw ingredients for life. But it also represents the abiotic chemical background against which signs of life must be identified, and should be understood in detail for its potential to yield false positive results. Complex and highly diverse suites of soluble organic compounds produced solely by non-biological processes have been found in meteorites and include amino acids, carboxylic acids, hydroxy acids, aldehydes, ketones, amines, N-heterocycles, aliphatic and aromatic hydrocarbons, sugars and other classes (Sephton, 2002) that collectively include millions of different possible chemical structures (Schmitt-Kopplin et al., 2010). A range of potential biosignatures occur within these same chemical classes, making it essential to characterize the molecular diversity within them in order to identify features that can be considered clear signs of life. For example, Terrestrial biology utilizes 20 standard, genetically encoded alpha-amino acids in proteins. Nineteen of these are chiral, meaning each amino acid can have two identical but non-superimposable ('left- and right-handed') molecular structures. Terrestrial life uses exclusively the left-handed forms (L-amino acids) in constructing its proteins - a selectivity thought necessary to confer proper function in biology - whereas laboratory experiments simulating plausible prebiotic conditions have produced chiral molecules in equal (racemic) mixtures of left and right-handed enantiomers (e.g. Miller, 1953; Yoshino et al., 1971). 'Homochirality' in amino acids and other compounds has thus been proposed as both a prevalent and relatively stringent indicator of life (e.g., Pace, 2001). Studies of astromaterials have proven essential in characterizing the abiotic prevalence of chiral excess in these compound classes. Ninety-six different amino acids have been identified and likely hundreds more detected in the Murchison meteorite (Glavin et al., 2018), and some meteorites exhibit L-amino acid excesses up to $60 \%$ and even higher excesses (to nearly 100\%) of D-sugar acids (Glavin et al. 2012; Cooper and Rios, 2016). This complicates the interpretation of chiral excess alone as a clear biosignature and emphasizes the necessity for contextual and complementary measurements in interpreting chiral excess as evidence of life.

Laboratory experiments designed to simulate the conditions and processes that occur in extraterrestrial environments - for example, cosmic ice irradiation and low temperature photochemistry - are an important complement to the characterization of astromaterials. These studies experimentally produce a wide range of compounds and features that add to our understanding of what is possible by abiotic means. For example, irradiation of ices containing comet-like mixtures of simple compounds produces a range of more complex compounds that are present in biological systems, including amino acids, quinones, and nucleic acids (e.g., Bernstein et al., 2002; Nuevo et al., 2009). These studies can also provide a mechanistic understanding of the processes involved, which allows the possibility to extrapolate results to conditions yet to be explored experimentally. 
It is essential that assessment of abiotic prevalence (background) be made for all proposed biosignatures. Characterization of astromaterials and complementary experimental approaches are critical in making this assessment.

\section{Technology}

The novelty of the measurements needed for life detection and the rigorous standards of evidence we will demand of them require new approaches in the conception, maturation, and deployment of instruments and instrument suites.

The nature and diversity of potential life detection measurements will require that we conceive sample analysis in end-to-end terms that encompass sample acquisition and handling, physical and/or chemical modification, 'front end' separation, and detection. Many life detection measurements will require ingestion and treatment - for example, via phase change, concentration, filtering, chemical derivatization, etc. - of samples whose physical and chemical properties are not well understood beforehand. Moreover, the chemical complexity of natural samples, even in the absence of life, can necessitate 'front end' separations that isolate specific targets or compound classes. Interpretation of results will require an understanding of how environmental context which may be poorly understood in advance - bears on the full end-to-end process of sample analysis. As one example, the Viking gas chromatography-mass spectrometry (GCMS) results were interpreted to indicate that the surface was devoid of organic compounds (Biemann et al., 1976). However, subsequent environmental characterization resulted in discoveries - e.g., widely distributed perchlorate salts (Hecht et al. 2009; Leshin et al. 2013) and unequivocal detection of organics (e.g. Freissinet et al. 2015) - that lead to reinterpretation of the Viking GCMS results a false negative resulting from the use of thermal sample volatilization (e.g., Navarro-Gonzalez et al., 2006, 2010; Guzman et al., 2018). Life detection measurements are thus not necessarily dependent on detection selectivity and sensitivity alone, but on end-to-end analytical considerations that must be understood in the specific context of environments in which measurements are made. It is critical that support for the development of life detection technologies encompass not just detection capability, but the end to end process of sample acquisition, treatment and analysis.

Life detection should involve correlated observations that represent independent assessments of biogenic character and provide necessary environmental context. Implementing a highly coordinated measurement strategy on planetary missions will require a novel approach to technology development. The traditional model of measurement technology and space instrument development has primarily focused on concepts proposed by individual teams, with measurement objectives naturally tied to the particular instrument type and team interest/expertise. Coordination of measurement approaches to address scientific objectives has been accomplished mainly at the mission level, generally well after the capabilities and requirements of each instrument are solidified. This model has produced decades of successful, ground-breaking investigations that have supported the objectives of planetary science effectively. To realize the level of coordination among measurements required for life detection objectives, NASA would strongly benefit from an expansion of the existing technology development model, to encourage collaborative development activities among research and technology teams. This will be accomplished most meaningfully if collaborative development begins at low TRL. In particular, the development of common sample processing and extraction "front-end" methods that have the potential to support multiple instruments would provide a functional basis for collaborative development, with the common goal of enabling complementary measurements to be performed on a single sample. 
Life detection methodology will require continuity with respect to past and future observations, the novelty to make measurements of a type not previously required, and the flexibility to accommodate the unanticipated. The need for correlation among measurements encompasses not just individual missions, but observations that may be spread across multiple missions. Environmental context is critical in interpreting potential evidence, or lack of evidence, for life. The challenges and pace of planetary exploration inherently limit the number of observations that can be brought to bear in establishing this context, so that measurements disconnected in space and time will have higher value if they can be nonetheless correlated through continuity of measurement approach. This may be especially important to consider in realizing the potential of competed mission classes to deliver high value life detection science, because the potential for limited observing capacity (relative to the larger payloads of flagship missions) could be compensated to an extent through correlation with measurements made by prior missions. At the same time, life detection will require measurements that are both novel relative to those typical of planetary exploration and also sufficiently flexible to accommodate unanticipated expressions of biological processes. To reconcile continuity, novelty, and flexibility in the development of measurement strategies and technology will require an unprecedented level of coordination between the researchers who identify and evaluate potential biosignatures and the technologists who develop and fly sample analysis systems. Creative means should be sought to foster the connectivity required between these communities, and we propose that joint engagement in developing a standard framework for biosignature assessment can be a useful starting point.

\section{Conclusion}

Ours could be the generation that discovers evidence of life beyond Earth. We should avail ourselves of the next well-conceived opportunity to seek this evidence and, in the interim, strive to do the groundwork that will allow us to make the most of it. We cannot control whether evidence of life lies within our reach, but we can control the means by which we seek it. A clear understanding of the challenges unique to life detection and robust investment in the suite of activities needed to address them will advance our readiness for this grand pursuit, informing our sense of where and how to look, and how to interpret what we find.

\section{References}

Bernstein, M., et al., (2002) Nature 416:401-403.

Biemann, K., et al., (1976) Science 194 (4260) : 72-76.

Cooper, G. \& Rios, A. C. (2016) Proc. Natl. Acad. Sci. USA 113 (24): E3322-E3331.

Freissinet, C. et al. (2015) J. Geophys. Res. 120 (3): 495-514.

Glavin, D. P., et al. (2012) Meteorit. Planet. Sci. 47 (8): 1347-1364.

Glavin D. P., et al. (2018) In Primitive meteorites and asteroids, Elsevier, pp. 205-271.

Hecht, M. H., et al. (2009) Science, 325 (5936): 64-67.

Miller, S. L. (1953) Science 117: 528-529.

National Academies of Sciences, Engineering, and Medicine (2019) An Astrobiology Strategy for the Search for Life in the Universe. The National Academies Press, Washington, D.C.

Nuevo, M., et al., (2009) Astrobiology: 683-695.

Pace, N. R. (2001) Proc. Natl. Acad. Sci. USA 98 (3): 805-808.

Schmitt-Kopplin P., et al. (2010) Proc. Natl. Acad. Sci. USA 107: 2763-2768.

Sephton M. A. (2002) Natural Product Reports 19: 292-311.

Yoshino, D., et al. (1971) Geochim Cosmochim Acta 35: 927-938. 